\title{
Polysèmes
}

Revue d'études intertextuelles et intermédiales

17 | 2017

L'art intempestif-La démesure du temps

\section{Le cycle in-fini chez Stuart Davis}

Stuart Davis: The In-finite Cycle

\section{Kamila Benayada}

\section{(2) OpenEdition}

Journals

Édition électronique

URL : http://journals.openedition.org/polysemes/1850

DOI : $10.4000 /$ polysemes. 1850

ISSN : 2496-4212

Éditeur

SAIT

\section{Référence électronique}

Kamila Benayada, «Le cycle in-fini chez Stuart Davis », Polysèmes [En ligne], 17 | 2017, mis en ligne le 30 avril 2017, consulté le 19 avril 2019. URL : http://journals.openedition.org/polysemes/1850 ; DOI : 10.4000/polysemes.1850

Ce document a été généré automatiquement le 19 avril 2019

Polysèmes 


\title{
Le cycle in-fini chez Stuart Davis
}

\author{
Stuart Davis: The In-finite Cycle
}

\section{Kamila Benayada}

1 La carrière de Stuart Davis, peintre américain né en 1892 et mort en 1964, recouvre une époque où les nouvelles technologies dans les transports ou les communications commencent à révolutionner le rapport au temps, en le contractant. Les découvertes en optique changent aussi la vision, elle-même affectée par le nouveau rapport au temps. Comme ses contemporains, les premiers modernistes américains qui se penchent sur ces innovations pour y trouver des sujets à peintre ${ }^{1}$, Davis s'intéresse à la télévision, à la radio, à l'aviation. En 1931, il peint une toile intitulée Television (collection privée), une autre intitulée Radio Tubes (Rose Art Museum, Brandeis Museum, Waltham, Massachusetts), puis en 1939, il propose une peinture murale sur l'histoire de l'aviation pour la foire mondiale de Flushing Meadow. Mais Davis s'intéresse moins à cette révolution technologique qu'à ses effets, notamment sur le temps et sa perception. Cependant, alors que Davis a intéressé les historiens de l'art dans sa relation au jazz ou à la publicité, ou dans sa relation au cubisme, son travail sur le temps n'a pas fait l'objet d'une étude détaillée. Pourtant, la relation des modernistes au temps est l'un des aspects fondamentaux de la rupture esthétique qui s'opère à la fin du XIX $\mathrm{X}^{\mathrm{e}}$ siècle, avec une recherche sur la non-linéarité de la représentation, et notamment son remplacement par les perspectives multiples ou la simultanéité, que Davis explore dans ses œuvres de jeunesse.

2 Une dizaine d'années avant sa mort, Davis se penche sur l'un des points essentiels de son esthétique, la continuité. En 1951, il peint une toile intitulée Visa, dans laquelle il inscrit la phrase "The/Amazing/Continu/ity »: l'étonnante continuité. La toile est l'une des œuvres d'une série commencée en 1950 (avec les différentes versions de Little Giant Still Life) et qui se poursuit jusqu'en 1961 (avec Schwitzki's Syntax). Il explique que cette continuité est une «forme à 5 dimensions $»^{2}$. Dans la même toile, il inscrit le mot «ELSE» (autre), et il indique dans ses notes sur une autre œuvre (American Painting) que ce mot, "else», est l'effaceur de la quatrième dimension: il efface, détruit ${ }^{3}$. "ELSE », l'autre, mais aussi l'effacement, est lié au principe de continuité. La quatrième dimension est à la fois celle du temps, pour certains (comme H.G. Wells), elle est aussi celle où la représentation des 
formes peut se faire dans de multiples directions. «ELSE » ouvre vers la possibilité de la différance, en retirant l'œuvre individuelle de la temporalité de la narration et du sujet (dans le sens de subject matter), et en en permettant la démultiplication infinie (errance).

3 Dans Visa, donc, Davis donne les principaux éléments des problèmes conceptuels qu'il tente de résoudre dans ses toiles : la question de la continuité, et celle de la différence, deux questions qui le mènent à une réflexion sur le temps, son intégration dans l'œuvre, et son dépassement. Son travail cyclique, où il reprend des toiles anciennes pour les retravailler, dans une sorte d'errance, est une tentative de trouver des solutions picturales à ces questions esthétiques et conceptuelles. Et ce travail passe par le dépassement du sujet (subject matter), de sa matérialité, de sa "réalité », puis par la répétition comme création, et la perception de l'œuvre comme mouvement ou durée, plutôt que comme moment/instant.

4 Ce travail et cette réflexion coïncident avec l'intérêt que portent les artistes des années 1960 au temps, à partir de la publication en 1962 de l'ouvrage de George Kubler, The Shape of Time: Remarks on the History of Things, ouvrage dans lequel l'auteur s'oppose à une histoire linéaire de l'art. Ad Reinhard publie en 1964 un compte rendu du livre de Kubler dans Art News ${ }^{4}$. Si je n'ai aucune preuve que Davis ait lu cet ouvrage, il est néanmoins intéressant de noter que cet ouvrage a marqué les artistes des années 1960, précisément du fait de son rejet du temps linéaire, que Davis rejette aussi, lui préférant la notion de continuité qui passe par la sérialité.

\section{Dépassement du sujet : vers une peinture an- historique}

Dans les années 1940, après avoir été un peintre engagé, Davis s'éloigne de la scène politique, et se lance dans une quête d'universalité et d'objectivité qui annule le sujet. En 1943, il écrit : « Aujourd'hui, je dissocie expérience de la peinture et expérience générale pour arriver à une expression universelle et objective où est transcendé l'aspect subjectif qu'il y a dans le particulier. L'émotion obtient alors une valeur intellectuelle au-delà du temps et de l'espace $»^{5}$. Il annonce ainsi sa rupture avec sa position des années 1930, son retrait de la scène politique, et il critique l'art engagé, subjectif, historique, lui préférant un art intellectuel, hors de l'espace-temps, sans lieu ou localisation précise. En 1952, il confirme ce qu'il entend par un art universel, qui est pour lui un art qui se dissocie du sujet : « on ne peut parler d'un art absolu, universel, que si l'on accepte de dissocier la peinture de l'expérience du sujet ou de l'état psychologique qui la précèdent $»^{6}$. Il y a donc rupture, et cette rupture se fait double : l'artiste renonce à son passé, et il renonce à s'intégrer dans le courant esthétique du moment (les débuts de ce qui sera appelé l'expressionnisme abstrait). Artiste « à rebours » (pour Elaine de Kooning), Davis n'est ni dans le passé, ni dans le présent ; il est dans un temps autre, anachronique.

6 Les années 1940 sont donc une période critique, charnière. Davis déclare renoncer au sujet, et revient à ce qui le préoccupait déjà dans les années 1920, où ses premières abstractions (notamment Matches, Percolator et la série Egg Beaters) réduisaient le sujet, $\mathrm{y}$ mettaient à nu, à travers des relations spatiales, la source physique de son impulsion ${ }^{7}$. Il s'agit donc déjà d'aller au-delà de la matérialité du sujet, mais Davis ne parle pas encore de le libérer de son historicité, de le libérer de lui-même, pour le libérer du temps (« beyond time »), ce qui deviendra l'un de ses objectifs. 
7 Ce processus de dépassement du sujet passe par l'exploration de relations binaires au sein d'une même toile, de deux ou même de plusieurs toiles. Dans les années 1920, Davis s'intéresse aux manifestations visuelles de la modernité, notamment à la vitesse et aux techniques cinématographiques. Cela le mène à explorer la question de la simultanéité, avec des toiles composées d'images juxtaposées, qui rappellent la succession d'images dans les pellicules cinématographiques ${ }^{8}$. Par exemple, dans House and Street (1931), Davis obtient deux effets contraires et complémentaires à la fois: d'une part il rompt la linéarité de la lecture avec la mise en relation de deux vues indépendantes, d'un côté un gros plan de maison, lui-même interrompu par le rectangle du bas où il inscrit le nom d'Al Smith (candidat démocrate à l'élection présidentielle en 1928), et de l'autre une rue; et d'autre part, le peintre produit un effet d'union de deux éléments disparates, joints dans un même plan noir, encadrés par une même bande rouge, qu'il coupe à gauche, par une ligne bleue. La rupture entre les deux parties est affirmée avec la ligne verticale du centre, mais cette même ligne reprend la ligne bleue du cadre, à gauche, puis l'échelle dans le gros plan de la maison, et Davis multiplie les verticales avec la cheminée à droite, puis le bord vert de la partie « rue ».

8 Il y a donc unité formelle et discontinuité narrative. La discontinuité narrative est poursuivie dans une version nouvelle de House and Street: The Mellow Pad (1945-51). Les deux toiles ont les mêmes dimensions, ce qui renforce l'effet de dédoublement. La maison devient un fond rouge, un arrière-plan pour une composition faite de juxtapositions et de superpositions. Les deux toiles coexistent dans la seconde version, Mellow Pad gardant les traces de House and Street, mais l'artiste détruit la maison et la vue de la rue en les transformant.

9 Ce travail de juxtaposition, sur la rupture et la continuité, réapparaît ailleurs, comme dans Deuce (1951-54), dont le titre ouvre la voie à une interprétation de ce travail conceptuel de l'artiste, alors que le titre House and Street, par exemple, pousse à une lecture littérale de l'œuvre, en nous faisant y rechercher plus l'anecdotique que le formel. Dans Deuce, comme dans House and Street ou Mellow Pad, Davis divise l'espace, juxtapose les formes, joue sur la dualité et sur la réversibilité : «deuce » indique l'égalité, ainsi que le chiffre deux (mais aussi le diable). Davis joue donc avec les doubles, les duos, la réversibilité. Il poursuit en 1961 avec Anyside, où la toile n'est pas composée de deux parties, mais où, comme le suggère le titre, la lecture est possible dans tous les sens. Le sens de lecture n'est donc pas clairement établi : la signature ne l'indique pas, car elle est autant lisible en haut de la toile que sur les côtés. Anyside supprime ainsi la linéarité de la lecture. L'artiste désordonne, dé-range, nous envoie dans des directions multiples. Cette indétermination de la lecture, c'est autant celle de la toile individuelle que de l'œuvre dans son ensemble. House and Street et Mellow Pad se succèdent dans le temps, mais peuvent être lues dans n'importe quel ordre.

10 Je reviens à House and Street et à la partie « maison » de la toile : Davis y inscrit le mot « FRONT », le devant, le premier plan, le début, qui appelle son contraire «back», l'envers, l'arrière, l'arrière-plan. Mais «back» ne figure pas dans la toile. "FRONT» réapparaît cependant dans la dernière toile de Davis, Fin (1964). Cette dernière toile reprend plusieurs œuvres antérieures (Punch Card Flutter, 1963, On location, 1963, Terrace, 1962, ou Bois, 1960), et Davis y fait basculer le dessin original, le renverse, le lit dans un autre sens. «FRONT », l'avant, le front, le début, alors, n'appelle pas son contraire, «back», puisque tout peut être «FRONT». Le sujet anecdotique, celui qui appelle une lecture temporelle, de gauche à droite, de haut en bas, du passé vers l'avenir, disparaît. Davis écrivait sur 
l'une des toiles de cette dernière série, Punch Card Flutter, «le mouvement répétitif des cartes perforées efface le sujet cliché " ${ }^{9}$. "Flutter», le battement, la palpitation, le mouvement répétitif qui va d'une œuvre à une autre efface le sujet. La répétition, le travail cyclique annule ainsi son historicité. Il permet l'exploration de directions multiples, les œuvres y étant des étapes : les formes réapparaissent à travers la carrière de Davis, dans de nouvelles configurations, avec de nouveaux titres, ou de nouvelles combinaisons de couleurs. La répétition vide le sujet et attire l'attention sur autre chose : la différence. La continuité, le retour constant du passé dans le présent, est ici non pas la répétition du même, mais l'occasion de faire rendre au sujet toutes les possibilités formelles qui sont en lui ; la continuité est la création elle-même.

\section{Peindre : l'éternel retour}

11 En 1930, Davis écrivait dans ses notes qu'une œuvre était complète, achevée, si elle faisait un emploi économe des réalisations du passé, pas en reproduisant le passé ${ }^{10}$. L'œuvre accomplie est reprise, mais elle implique une transformation, elle n'est pas répétition à l'identique. Davis écrit au sujet de cette continuité, sur laquelle il attire l'attention dans Visa, que cette étonnante continuité n'est pas similarité, mais au contraire elle est le dissemblable ${ }^{11}$. Cette continuité, donc, introduit non pas la similitude, mais un système de différences. L'ensemble des peintures de Davis constitue un "espace de coexistence " (Deleuze 1), d'« éléments différentiels » et similaires à la fois, ce qui crée un système de relations.

Les formes, comme les mots, chez Davis, reviennent, se chevauchent et s'imbriquent au sein des toiles et d'une toile à l'autre. Le cycle n'est jamais fermé. La toile est toujours un champ de migration, un point d'entrée et de sortie. Dans le croquis préparatoire de sa dernière toile, Davis écrit en haut à gauche, sous le mot " front ", « in out field » : la toile est un champ où les éléments entrent et sortent, circulent dans un mouvement infini. De plus en plus, Davis peint dans un système clos, un circuit fermé (Closed Circuit, 1962). La nouveauté vient du passé. Davis introduit le mot «New» dans plusieurs toiles : la série Package Deal, Premiere, et International Surface (1956-60), et dans les deux versions de Combination Concrete (1958). Les éléments qui constituent Package Deal, peint en 1956, sont repris dans plusieurs autres toiles: Package Deal devient Premiere puis International Surface ; les trois toiles contiennent le mot «New», qui disparait dans les versions suivantes, Standard Brand (1961) et Blips and Ifs (1963-64). " New » apparaît aussi dans Combination Concrete qui est un réassemblage de toiles anciennes, les premières étant des paysages datant de 1922 : Landscape, Gloucester et Landscape. La nouveauté est créée par la rupture avec le présent, elle vient du re-travail du passé, du réassemblage, de la combinaison différente de formes trouvées dans son propre travail. Intericonicité, allées et venues, chevauchement, pluralité des temporalités, les toiles de Davis forment un ensemble instable, perméable, où le passé se superpose au présent, et préfigure des œuvres à venir.

Dans Owh! in San Pao (1951), Davis écrit : « used to be - NOW : « était - MAINTENANT ». Ce qui était, est, dans le présent. Cette toile reprend Percolator (1927), sans grandes variations hormis dans la palette et l'addition des inscriptions, « used to be - NOW » à droite et «ELSE » à gauche. La nouvelle version efface la précédente («ELSE») tout en la gardant (« used to be »). Ruptures et continuités dans la lecture et dans le temps sont dédoublées : Davis introduit des ruptures et continuités formelles en organisant l'espace de sorte que ses constructions picturales emmènent l'œil vers l'arrière (back) pour le ramener vers l'avant 
(front). Les plans dans Percolator et Owh! in San Pao s'entrechoquent, la profondeur est à la fois affirmée (avec les plans en diagonale) et annulée (avec les aplats). En 1956, il note son intérêt pour l'opposition dialectique entre avant et arrière-plans ${ }^{12}$, intérêt qui apparait ailleurs dans ses écrits : « La présence de deux systèmes spatiaux en opposition [...] établit une relation de plans spécifique. [...] Les éléments dialectiques, dont l'appréhension simultanée et contradictoire crée une forme esthétique, sont les suivants : longueur, plan, couleur-ton $»^{13}$. L'expérience esthétique provient de cette opposition simultanée entre les différents plans. Elle provient également de l'expérience simultanée des œuvres dans le temps. De la même manière que les plans se chevauchent et s'enchevêtrent, les toiles se mêlent, résistent à la linéarité du temps, car le passé et le présent y coexistent. La toile n'est plus dans le temps, au présent ou au passé, elle ouvre vers des temps et des espaces démultipliés. Ce qui était « front » dans House and Street, le premier plan, devient l'arrièreplan de Mellow Pad. La perspective traditionnelle est rompue, l'horizon spatial et temporel est instable. Le temps et l'espace se déplacent d'une toile à l'autre (Davis écrit dans ses notes sur Owh! in San Pao qu'un déplacement du temps et de l'espace avait eu lieu alors qu'il travaillait $\left.{ }^{14}\right)$. «Front » devient «back», et peut redevenir « front ». Espace et temps fonctionnent ensemble; le travail sur le premier plan et l'arrière-plan est aussi un travail sur le passé et le présent.

Le double détruit le sujet; il détruit également le temps en annulant la différence entre passé et présent. Les toiles existent sur le même plan : à la fois anciennes et nouvelles, finies et incomplètes, voire in-finies. Toute toile est Unfinished Business (1962), titre qu'il donne à une peinture où il reprend le mot « $P A D$ » (qui vient de la toile Mellow Pad) et où la structure est encore une fois binaire. Comme précédemment, gauche et droite sont réversibles, les lettres à gauche ("ONO ») sont un palindrome, la lecture elle-même est infinie, car elle part d'autres toiles et doit se poursuivre dans des toiles à venir (dans ce cas, avec un travail sur la couleur, où Davis reprend la même palette, dans les toiles de la série débutée en 1960 avec Bois).

À travers la répétition, Davis déplace l'acte de peindre vers la création d'un espace en mouvement perpétuel. La toile Closed Circuit l'indique bien : en peignant en circuit fermé, Davis crée une dynamique semblable à celle du courant électrique infini, qui est infini du moment que le circuit reste fermé. En même temps, la répétition dans la différence est aporétique : la dynamique infinie indique une expérience qui ne peut/ne doit pas aboutir, qui est en suspens, en attente. Le présent est démultiplié, prolongé, comme le passé, vers un avenir toujours repoussé dans le temps.

\section{Durées}

L'œuvre chez Davis est processus, elle est projet: en errance perpétuelle, le peintre avance sans jamais arriver à une destination quelconque. Dans le croquis préparatoire pour la gouache Bois, il écrit en haut : «Method - Content - Simultaneous»: Méthode Contenu - Simultané. Méthode et contenu, forme et sujet, sont simultanés, indissociables dans le temps. Mais le sujet est déplacé, il devient le processus créatif lui-même ${ }^{15}$. Les inscriptions sur les toiles sont des indications conceptuelles, des renvois à ses notes où il élabore son esthétique. La toile est le lieu de visualisation de la pensée en mouvement, où l'artiste met au point différentes solutions picturales à des problèmes théoriques esthétiques. Et ce processus introduit la durée dans la continuité. La perception de la continuité qu'a Davis a quelque chose de bergsonien, car elle inclut le changement et le 
passé : pour lui comme pour Bergson, « la durée est le progrès continu du passé qui ronge l'avenir et qui gonfle en avançant » (Bergson 4). Il y a interaction entre passé, présent, et avenir. Les formes viennent du passé, et subissent l'effet de la réflexion présente, qui est une piste à suivre dans le futur. Le passé est anticipé, les toiles vont au-delà de lui, le dépassent. Les toiles sont liées, d'une part par la réutilisation de formes, mais aussi par la mise en œuvre des théories de l'artiste. Le circuit fermé place les œuvres et le travail de Davis hors du temps, dans un temps interne continu et infini. Davis écrit en 1952 que l'art est un ensemble de maintenants compris dans la dimension de la continuité qui les rend tous égaux ${ }^{16}$. Le principe de la continuité annule le temps en ce qu'elle efface le présent ( NOW) de la même manière que l'art efface le sujet (subject matter) en l'incluant dans le processus créatif.

Mais la continuité est faite de moments qui sont indépendants autant qu'ils sont liés. Chaque toile peut être lue seule; et le plus souvent Davis ne reprend pas les titres originaux dans les nouvelles versions de ses œuvres. Et à travers ces nouveaux titres, il y a mise à distance : l'artiste rompt avec le passé, fragmente la lecture. Si la durée est liaison, l'œuvre de Davis crée la durée et la détruit en même temps. Le travail inaccompli ("Unfinished Business ») est repris plus tard, ailleurs, dans d'autres toiles, mais ce travail est, par la force des choses, fragmentaire. Il est disséminé de toile en toile. Les formes sont « semées » à travers son œuvre : en 1953, Davis peint Semé, où il reprend Landscape, Gloucester de 1922, qui avait déjà été le point de départ de Rapt at Rappaport's (1952). Dans un article de 1953 sur Rapt at Rappaport's, Davis explique qu'en regardant Landscape, Gloucester, une réaction en chaîne s'est déclenchée, les formes initiales suggérant de nouvelles associations ${ }^{17}$. Les toiles ainsi reliées à Landscape Gloucester (dont Combination Concrete évoquée plus haut) constituent moins un cycle, qui serait fermé, qu'un enchaînement. De la même manière que, dans le titre de la toile de 1952, « Rapt » semble engendrer "Rappaport » (les deux ont la même racine, les mêmes consonnes), une toile engendre tout un ensemble d'autres toiles qui à leur tour en engendrent d'autres. L'œuvre individuelle en appelle d'autres, en attend d'autres, est à la frontière, au bord d'une nouvelle œuvre, elle est création en suspens. D'une certaine manière, chaque œuvre est une avancée vers la proximité du lointain (ce que Heidegger explique dans Être et Temps $)^{18}$. Chaque œuvre est la résolution temporaire d'un problème esthétique; elle s'approche du but, tout en l'éloignant, en le repoussant, car l'artiste y découvre autre chose à explorer, autre chose en latence, le «non découvert $»^{19}$; l'achèvement est perpétuellement différé. Davis interroge les limites de la forme, en en démultipliant les possibilités, mais aussi en montrant l'impossibilité de cerner complètement l'image latente que la forme contient. Si les formes en appellent d'autres, c'est peut-être qu'il y a en elles, ou plutôt dans le sujet que Davis détruit dans la répétition, la présence d'une absence que l'exploration formelle tente de combler, tout en repoussant indéfiniment la chute.

18 Quand, en 1914, Davis décide de devenir un artiste "moderne ", il entame un travail sur le rapport au temps, le menant de la simultanéité (avec les changements dans la perception qui accompagnent la révolution dans les transports : vitesse, accélération, vision panoramique), à travers une étude du cubisme, puis à la continuité, de l'effacement du passé à son anticipation infinie. Le travail cyclique chez Davis, la sérialité, met en œuvre une dynamique en mouvement perpétuel, mais la durée que cette dynamique instaure est également latence, report, attente. Davis, en cela annonce les questions sur le temps que se poseront les artistes des années 1960. Le système qu'il crée à travers la 
sérialité est un système cybernétique, dans la mesure où il n'est pas fermé, qu'il exige d'être nourri constamment, de façon infinie ${ }^{20}$. Les effets de miroir, les dédoublements, les échos (Rapt at Rappapport's), ces jeux de variations et de travestissements du sujet, ces fusions et confusions formelles et temporelles sont les bases d'une esthétique complexe fondée sur le report, le suspens, où le familier, ce que l'œil reconnaît (le « lisible », mais aussi les autocitations), tout ceci est brouillé, renversé, pour en détourner le sens, le soustraire à notre appréhension, nous emmener encore ailleurs, dans d'autres toiles. L'œuvre en mouvement ne trouve sa résolution que dans la toile ultime de Davis, Fin, qui met un point final au mouvement créatif, à l'œuvre en projet. La dynamique « in-out » est interrompue, le circuit désormais ouvert. L'expérience aporétique n'est terminée que par la mort du peintre. L'artiste, finalement, est rattrapé par le temps.

\section{BIBLIOGRAPHIE}

\section{Textes cités}

Bergson, Henri. L'Évolution créatrice (1969). Paris : Quadrige/PUF, 1981.

Deleuze, Gilles. Différence et répétition (1968). Paris : PUF, 2005.

Heidegger, Martin. Being and Time. Trans. John Macquarrie \& Edward Robinson. New York: Harper and Row, 1962.

Heidegger, Martin. Poetry, Language, Thought. Trans. Albert Hofstadter. New York: Harper and Row, 1971.

Kelder, Diane (ed.). Stuart Davis. New York, Washington and London: Praeger, 1971.

Lee, Pamela M. Chronophobia: On Time in the Art of the 1960s. Cambridge, Mass. and London: MIT

Press, 2004.

Sims, L.S. (ed.). Stuart Davis, American Painter. New York: The Metropolitan Museum of Art/Harry N. Abrams, 1991.

Sweeney, J.J. Stuart Davis. New York: MoMA, 1945.

\section{Tableaux de Stuart Davis cités}

American Painting (1932-51). Huile sur toile. Joslyn Art Museum, Omaha, University of Nebraska at Omaha.

Anyside (1961). Huile sur toile. Collection privée.

Blips and Ifs (1963-64). Huile sur toile. Amon Carter Museum, Fort Worth.

Bois (1960). Gouache sur papier. Collection Jonas Aarons, South Orange, New Jersey.

Closed Circuit (1962). Caséine sur papier. Collection privée. 
Combination Concrete (1958). Huile sur toile. Collection privée.

Deuce (1951-4). Huile sur toile. San Fransisco Museum of Modern Art.

Fin (1964). Huile, caséine, encaustique et ruban adhésif sur toile. Collection Earl Davis.

House and Street (1931). Huile sur toile. Whitney Museum of American Art, New York.

Landscape (1922). Huile sur panneau. Collection Earl Davis.

Landscape, Gloucester (1922). Huile sur panneau. Collection Phyllis et Jerome Lyle Rapapport.

Little Giant Still Life (1950). Huile sur toile. Virginia Museum of Fine Art, Richmond.

Matches (1927). Huile sur toile. Chrysler Museum, Norfolk, Virginie.

On Location (1963). Caséine et mine de plomb sur papier. Collection Earl Davis.

Owh! In San Pao (1951). Huile sur toile. Whitney Museum of American Art.

Package Deal (1956). Gouache sur papier. Collection privée.

Percolator (1927). Huile sur toile. Metropolitan Museum of Art, New York.

Première (1957). Huile sur toile. National Museum of American Art, Smithsonian Institution,

Washington D.C.

Punch Card Flutter (1963). Huile sur toile. Collection Earl Davis.

Rapt at Rappaport's (1952). Huile sur toile. Hirshhorn Museum, and Sculpture Garden, Smithsonian Institution, Washington D.C.

Schwitzki's Syntax (1961). Huile, ruban adhésif et peinture à l'encaustique. Yale University Art Gallery, New Haven.

Semé (1953). Huile sur toile. Metropolitan Museum of Art, New York.

Standard Brand (1961). Huile sur toile. Detroit Institute of Arts.

Terrace (1962). Huile sur panneau. San Diego Museum of Art.

The Mellow Pad (1945-51). Huile sur toile. Collection Edith et Milton Lowenthal, New York.

Unfinished Business (1962). Huile sur toile. Collection William H. Lane.

Visa (1951). Huile sur toile. MoMA, New York.

\section{NOTES}

1. Voir par exemple Linda Dalrymple Henderson : "X-Rays and the Quest for Invisible Reality in the Art of Kupka, Duchamp, and the Cubists", Art Journal (Winter 1988): 323-340, Robert Mark Antliff: "Bergson and Cubism, a Reassesment", Art Journal (Winter 1988), ou encore Gordon Hughes: "Envisioning Abstraction: The Simultaneity of Robert Delaunay's First Disk", The Art Bulletin, 89.2 (June 2007): 306-332. Sur l'aversion au temps chez les post-modernes des années 1960, voir Pamela M. Lee, Chronophobia, on Time in the Art of the 1960s, Cambridge, Mass. and London: MIT Press, 2004.

2. "A shape of 5 dimensions ». Cité in Lewis Kachur : "Stuart Davis's Word Pictures", Stuart Davis, American Painter, ed. Lowery Stokes Sims, New York: The Metropolitan Museum of Art/Harry N. Abrams, 1991, 102.

3. «Else is the $4^{\text {th }}$ dimensional Eraser » (Sims 102). 
4. Voir Pamela M. Lee, Chronophobia: On Time in the Art of the 1960s, Cambridge, Mass. and London: MIT Press, 2004, notamment dans son chapitre 4.

5. "Today, I disassociate [sic] the painting experience from general experience and attain a universal and objective statement that transcends the subjectively particular. The emotion is given an intellectual currency beyond time and place", Stuart Davis Papers (SDP), July 1943, Harvard Art Museums, Fogg Art Museum, Cambridge, Mass.

6. «When it is understood that painting is completely disassociated from the experience in subject matter and psychological attitude that preceded it, it becomes possible to talk about an absolute, universal art » : déclaration à Alfred H. Barr, Jr., 3 nov. 1952, à l'occasion de l'acquisition par le MoMA de Visa (1951), reproduit dans Stuart Davis, ed. Diane Kelder, New York, Washington, and London: Praeger, 1971, 102. Cette déclaration est une prise de position qui place Davis aux marges du champ artistique car l'artiste se prononce contre les expressionnistes abstraits et la définition de l'art donnée par Clement Greenberg.

7. « ... strip the subject down to the real physical source of its stimulus. I felt that a subject has its emotional reality fundamentally through our awareness of [...] planes and their spatial relationship ", Davis, au sujet de la série Egg Beaters, cité dans J.J. Sweeney, Stuart Davis, New York: MoMA, 1945, 17.

8. En 1940, Davis fait la liste des choses (objets ?) qui avaient inspiré sa toile Hot Still Scape for Six Colors - Seventh Avenue Style: «neon tubes, music through radio, motion picture juxtapositions, skywriting, etc...» (SDP, 2 sept. 1940).

9. « Punch Card Flutter Erases Cliché Subject », SDP, 22 mai 1963.

10. "Completion must be understood as the economical use of past accomplishment, not a redoing of it », SDP, 25 mars 1931.

11. "The Amazing Continuity is not an order of likeness, but a system of Unlikeness ", SDP, 1 nov. 1951.

12. « This Front-Back position is dialectical », SDP, 8 juin 1956.

13. «The presence of two opposing space systems [...] establish(es) a specific planal relationship. [...] The dialectical elements, whose contradictory simultaneous experience creates the aesthetic form, are as follows: length, area, color-tone » (cité dans Sims 60).

14. "Great displacement of Time-Space took place as [I] work on [the painting] », Stuart Davis Calendar, 19 août 1951, archives de Earl Davis (cité dans Sims 276).

15. W.C. Agee, «Stuart Davis in the 1960s, the Amazing Continuity (Sims 92).

16. "Art is Nows $[s i c]$ in the Dimension of Continuity which makes all Nows Equal », SDP, 2 août 1952.

17. «[It] set off a chain reaction [...] as these initial shapes and diversions [suggested] new associations », D.G. Seckler, « Stuart Davis Paints a Picture », Art news 52.4 (Summer 1953): 31.

18. "A coming into the nearness of distance", Martin Heidegger, Being and Time, trans. John Macquarrie \& Edward Robinson, New York: Harper and Row, 1962, 203-210.

19. Heidegger écrit dans "The Origin of the Work of Art »: "Truth is an un-truth, insofar as there belongs to it the reservoir of the not-yet-uncovered, the un-uncovered, in the sense of concealment ", Poetry, Language, Thought, trans. Albert Hofstadter, New York: Harper and Row, 1971, 60-61.

20. Pamela M. Lee écrit : "A cybernetic system is not a closed entity but one that is open to, indeed, demands feedback ». «A cybernetic system folds together the past, present, and future in a continuous loop; it is potentially endless ", Chronophobia: on Time in the Art of the 1960s, Cambridge, Mass. and London: MIT Press, 2004. 


\section{RÉSUMÉS}

Les œuvres sérielles du peintre américain Stuart Davis sont un travail sur la simultanéité, la durée, la continuité, autant que sur la différence. J'analyse ici les œuvres que Davis produit après 1940, dans leur relation les unes aux autres, et aux œuvres de jeunesse. Prises dans un circuit fermé, les œuvres superposent passé, présent, et annoncent le futur, offrant une expérience simultanée de temps multiples. La continuité qui se produit ainsi est le principe même de la création, qui est recherche perpétuelle, et mouvement ininterrompu, in-fini, autant qu'elle est latence, attente, report.

American painter Stuart Davis's serial work explores simultaneousness, duration, continuity, as well as/through difference. In this article, I look at Davis's post-World War 2 works in their relation to one another and to earlier works. Davis painted in a "closed-circuit" in which past, present and future are superimposed, offering a simultaneous experience of multiple times. The continuity that is thus produced is the principle of creation in Davis's work, which is research in process, uninterrupted, unending and infinite movement as much as it is latency, delay, and expectation.

\section{INDEX}

Keywords : simultaneousness, an-historicity, continuity, seriality, difference, movement Mots-clés : simultanéité, an-historicité, continuité, sérialité, différence, mouvement

\section{AUTEURS}

\section{KAMILA BENAYADA}

Kamila Benayada est maître de conférences en études américaines à l'université d'Orléans. Son domaine de recherche est l'histoire et analyse de l'art des États-Unis au XX $\mathrm{X}^{\mathrm{e}}$ siècle, en particulier l'œuvre de Stuart Davis. Elle a publié dans RFEA, LISA, Transatlantica, Textes et Contextes, Interfaces. Elle est co-auteur de L'Amérique des Images, histoire et analyse des images dans la culture américaine, dir. François Brunet (Paris : Hazan, 2013), Art et Utopie, dir. Mathilde Arrivé (Paris : Michel Houdiard, 2012). 